\title{
Friedrichs II. Montezuma (1755): Ein aztekischer Anti-Machiavel
}

\section{Achim Aurnbammer}

Die spanische Eroberung Mexikos unter Hernán Cortés beschäftigte die europäische Imagination seit ihrer Anklage durch Bartolomé de Las Casas' Historia general de las Indias (1552). Getragen wurde die Kritik an Cortés und seiner Vernichtung des Aztekenreichs zum einen von grundsätzlichen Bedenken gegenüber der Behandlung amerikanischer Ureinwohner, zum anderen von einem anti-spanischen Ressentiment, das durch politische Ängste und konfessionelle Vorbehalte geschürt wurde. Insbesondere auf den protestantischen Bühnen des 17. und frühen 18. Jahrhunderts erfreute sich der Conquista-Stoff daher einiger Beliebtheit, auch weil er die Möglichkeit bot, den Kampf der Azteken gegen die Spanier nach Mode der Zeit mit exotischem Kolorit auszuschmücken. William Davenants Maskerade The Cruelty of the Spaniards in Peru (1658, Musik von Matthew Locke) behandelt vorrangig die Eroberung Perus, inspirierte aber John Drydens The Indian Emperour (1665), das den dramatischen Konflikt zwischen Montezuma und Cortés in den Mittelpunkt stellt und den Aztekenkönig erstmals als edel und stolz porträtiert. Das Schwesterstück The Indian Queen (1664), von Dryden gemeinsam mit Richard Howard verfasst, wurde 1695 von Henry Purcell zur Semi-Oper erweitert. Im Zuge der Aufklärung wuchs die Faszination für die amerikanische Conquista weiter an. Wenngleich Alexis Pirons Tragödie Fernand Cortez où Montezume (1744) versucht, die spanische Eroberung zu rehabilitieren, blieb der Grundtenor kritisch. Bereits in Bernard de Fontenelles Dialogues des Morts (1683) entspinnt sich eine Diskussion zwischen Cortés und Montezuma, in welcher der Azteke mit der Stimme der Vernunft und Menschlichkeit spricht und das zivilisatorische Überlegenheitsdenken der Europäer anprangert.

Womöglich angeregt durch Fontenelles Dialogue, gestaltete auch König Friedrich II. von Preußen das Motiv einer Begegnung der Alten und der Neuen Welt. ${ }^{1}$ In seiner Tragödie Montezuma wird der Titelheld als guter Fürst präsentiert, während der spanische Konquistador eben jene machiavellistische Herrschaftsauffassung vertritt, die Friedrich II. in seinem Traktat Anti-Machiavel (1740) zu widerlegen suchte. Friedrich verfasste seine Azteken-Tragödie in französischer Sprache, sein italienischer Hofdichter Giovan Pietro Tagliazucchi übertrug sie in ein ita-

1 Friedrich II. besaß Fontenelles Werke in verschiedenen Ausgaben; siehe Bogdan Krieger: Friedrich der Große und seine Bücher. Leipzig 1914, 160. 1773 verfasste der König selbst ein Totengespräch, nämlich den Dialogue des morts entre Madame de Pompadour et la Vierge Marie. Siehe Friedrich II.: Totengespräch zwischen Madame de Pompadour und der Jungfrau Maria, hg., übers. und kommentiert von Gerhard Knoll. Berlin ${ }^{2} 2000$. 
lienisches Libretto, ${ }^{2}$ das der Hofkapellmeister Carl Heinrich Graun vertonte. ${ }^{3}$ Am 6. Januar 1755 wurde in der Königlichen Oper Unter den Linden in Berlin Montezuma als Tragedia per musica uraufgeführt. Zur Uraufführung erschien ein italienisch-deutsches Textbuch in Paralleldruck; wer die zeilengerechte deutsche Übersetzung verfasst hat, ist unbekannt. Nach sechs Wiederholungen im Januar 1755 ging die Opera seria erst wieder nach dem Siebenjährigen Krieg zum Karneval 1771 in Szene, wozu das italienisch-deutsche Textbuch fast unverändert neu gedruckt wurde. ${ }^{4}$

\section{Forschung}

Friedrichs II. Montezuma ist ebenso gut erforscht wie die musikalische Gestaltung durch Carl Heinrich Graun. Das Bühnenwerk gilt als beachtlicher „Vorläufer der Gluck'schen Reform-Oper“. ${ }^{5}$ Die Berliner Uraufführung ist zum 250. Jubiläum

2 Vgl. Anon. [Friedrich II., Kg. von Preußen]: Montezuma. Tragédie en trois actes (mit frz. Vorrede von [Giovan Pietro] Tagliazucchi). Berlin (J. G. Michaelis) o. J. [1755]. Auf die Autorschaft Friedrichs des Großen wird antonomastisch, aber unmissverständlich hingewiesen: „La Poësie est l'ouvrage d'un Esprit sublime“ (7). Das italienische Libretto mit anonymer zeilengetreuer Übersetzung erschien unter folgendem Titel: Anon. [Giovan Pietro Tagliazucchi]: Montezuma. Tragedia per musica da rappresentarsi nel Regio Teatro di Berlino per ordine di Suà Maestà il Re nel Carnovale del MDCCLV. / Montezuma[,] ein musicalisches Tranerspiel[,] welches auf Sr. Königl. Maj. In Preussen allergnedigsten Befehl auf dem Berlinischen Schauplatz währendem Carneval des 1755sten Jahrs aufgefübrt werden soll. Berlin (Haude und Spener) o. J. [1755]. Auf diese Ausgabe beziehen sich im folgenden die eingeklammerten Akt-, Szenen- und Seitenzahlen im Text. Die metrische Struktur von Tagliazucchis italienischer Version blieb bislang unbehandelt. In den sangbaren Versi sciolti der Rezitative wechseln meist Endecasillabi mit Settenari ab. Reime kommen nur als Paarreime vor, die reimlosen Verse dominieren. Die fast durchgängig in Settenari abgefassten zweiteiligen Arien beziehungsweise Kavatinen weisen überwiegend Reimverse mit komplexeren Reimformen auf, aber auch zahlreiche Waisen. Diese freie Reimbehandlung rechtfertigt Tagliazucchi unter Berufung auf „Despreaux“, gemeint ist Nicolas BoileauDespréaux (1636-1711), der maßgebliche Theoretiker des französischen Klassizismus.

3 Zu Graun vgl. Christoph Henzel: Graun, Carl Heinrich. In: Musik in Geschichte und Gegenwart, Personenteil, Bd. 7. Kassel [u.a.] 22002, 1506-1525, sowie Hans-Joachim Bauer: Carl Heinrich Graun. In: Carl Dahlhaus und das Forschungsinstitut der Universität Bayreuth (Hg.): Pipers Enzyklopädie des Musiktheaters. Oper. Operette. Musical. Ballett, Bd. 2. München und Zürich 1987, 555-560, zu Montezuma besonders 557-560.

4 Vgl. Anon. [Giovan Pietro Tagliazucchi]: Montezuma. Tragedia per musica da rappresentarsi nel Regio Teatro di Berlino per ordine di Sua Maesta il Re nel Carnovale del MDCCLXXI. / Montezuma, ein musicalisches Tranerspiel welches, auf Sr. Königl. Maj. in Preussen allergnedigsten Befehl auf dem Berlinischen Schauplatze, währendem Carneval des 1771sten Jahrs aufgeführt werden soll. Berlin (Haude und Spener) 1771. Vgl. die bibliografisch exakte Aufstellung von Reinhart Meyer (Hg.): Bibliographia dramatica et dramaticorum, 2. Abt.: Einzeltitel, Bd. 18 (17551757). Tübingen 2003, s. v. Tagliazucchi, Giampietro (Jean Pierre), 182-185.

5 Vgl. das Vorwort des Herausgebers in Carl Heinrich Graun: Montezuma, hg. von Albert Mayer-Reinach (Denkmäler deutscher Tonkunst 1, 15). Wiesbaden und Graz 1958 [Leipzig 1904], V-XXIII, hier XI. Knappe, aber eindringliche Musikanalysen liefert Nadège Bourgeon: La figure du conquérant dans l'opéra 'Montezuma' de Carl-Heinrich Graun et Frédéric II de Prusse. In: Irène Mamczarz (Hg.): Le héros légendaire sur les scènes du théâtre et de l'opéra. Florenz 2001, 259-266. 
neu inszeniert sowie in einer eigenen Ausstellung und einem wissenschaftlichen Symposium gewürdigt worden. ${ }^{6}$ Die Forschung hat die Personenkonstellation des Montezuma mit Friedrichs Machiavelli-Kritik parallelisiert, ${ }^{7}$ die Idealisierung des Aztekenkaisers mit der Sympathie eines aufgeklärten Monarchen erläutert ${ }^{8}$ und die Abwertung des Hernán Cortés ${ }^{9}$ mit der sogenannten leyenda negra erklärt, derzufolge Spanien im 18. Jahrhundert allgemein als Hort der Gegenaufklärung galt. Schließlich wurde der Montezuma sowohl auf seinen historischen Kern als auch motiv- und diskursgeschichtlich auf die Literarisierungen der Begegnung von Alter und Neuer Welt bezogen. ${ }^{10}$ Dennoch ist manches ungeklärt: Strittig blieb etwa die

6 Vgl. dazu Ruth Müller-Lindenberg (Hg.): Friedrichs Montezuma. Macht und Sinne in der preuBischen Hofoper (26.1.-24.6.2012). Publikation zur Sonderausstellung im MusikinstrumentenMuseum des Staatlichen Instituts für Musikforschung Preußischer Kulturbesitz. Berlin 2012. Vgl. die Online-Publikation unter http://friedrich-montezuma.de, 29. März 2016.

7 Vgl. Arndt Krieger: Moral und Geschichte: Die Oper ,Montezuma' von Friedrich dem Großen. In: Wilhelm G. Busse und Olaf Templin (Hg.): Der einsame Held. Tübingen und Basel 2000, 125-145.

8 Vgl. etwa Heinz Klüppelholz: Die Eroberung Mexikos aus preußischer Sicht. Zum Libretto der Oper ,Montezuma' von Friedrich dem Großen. In: Albert Gier (Hg.): Oper als Text. Romanistische Beiträge zur Libretto-Forschung (Studia Romanica; 63). Heidelberg 1986, 65-94. Er erklärt in seinem close reading die angeblich „flächige Figurenkonzeption“ und ,aufklärerische Thesenhaftigkeit“ (92) mit einer gewissen Distanz Friedrichs, der dem idealisierten ,edlen Wilden' doch nicht "die gleiche in der Natur des Menschen begründete Vernunft zuerkennen [wolle] wie sich selbst" (94).

9 Aus Gründen der Einheitlichkeit markiert die Schreibweise, Cortés` hier durchgängig die historische Person, während für die Bühnenfigur die Schreibweise ,Cortes ' aus Friedrichs Montezuma übernommen wird.

10 Heinz Klüppelholz (Anm. 8) rekonstruiert umständlich die Ereignisgeschichte von Cortés' Eroberung und legt seiner Studie weder das historische Libretto noch die Edition der Partitur zugrunde, sondern eine aktualisierte Strichfassung, die Herbert Quander im Jahre 1981 für Herbert Wernickes Inszenierung des Montezuma im Berliner Hebbel-Theater angefertigt hat. Vgl. dazu auch die diversen, sich stark überschneidenden und repetitiven Studien von Jürgen Maehder, die alle von Tzvetan Todorovs Bestimmung der Entdeckung der Neuen Welt als der Entdeckung des Anderen überhaupt ausgehen: Jürgen Maehder: The Representation of the ,Discovery' on the Opera Stage. In: Carol E. Robertson (Hg.): Musical Repercussions of 1492. Encounters in Text and Performance. Washington und London 1992, 257-279, wo mit breiten Zitaten der Wandel des Amerikabildes dokumentiert wird. Jürgen Maehder: Mentalitätskonflikt und Fürstenpflicht. Die Begegnung von mittelamerikanischem Herrscher und Conquistadór auf der barocken Opernbühne. In: Michael Walter (Hg.): Text und Musik. Neue Perspektiven der Theorie (Materialität der Zeichen A; 10). München 1992, 131-179. Etwas präziser gefasst in: Jürgen Maehder: Die Opfer der Conquista. Das Volk der Azteken auf der Opernbühne. In: Peter Csobádi [u.a.] (Hg.): ,Weine, weine, du armes Volk'. Das verfübrte und betrogene Volk auf der Bübne, Bd. 1 (Wort und Musik; 28). Anif und Salzburg 1995, 265-287 (mit einem Anhang der Kolumbus- und Montezuma-Opern zwischen 1600 und 1992). Jürgen Maehder: Ein königliches Libretto. Probleme der Librettoforschung, dargestellt am Montezuma-Libretto Friedrichs des Großen. In: Udo Bermbach und Wulf Konold (Hg.): Gesungene Welten. Aspekte der Oper (Oper als Spiegel gesellschaftlicher Veränderungen; 2 / Hamburger Beiträge zur öffentlichen Wissenschaft; 10). Berlin und Hamburg 1992 (mit einem Verzeichnis von Kolumbus- und Cortés-Opern). Jürgen Maehder: Cristóbal Colón, Motecuzoma II. Xocoyotzin and Hernán Cortés on the Opera Stage A Study in Comparative Libretto History. In: Revista de Musicología 16, 1993, 146-184 (mit einer bibliografischen Präzisierung der Liste der Kolumbus- und Cortés-Opern). 
literarhistorische Bedeutung von Friedrichs Tragedia per musica. So wurde wegen mancher sprachlicher Parallelen Voltaires Tragödie Alzire, ou les américains als Prätext angeführt. ${ }^{11}$ Allerdings ist die Strukturanalogie, die in der Konstellation der edlen Inkaprinzessin Alzire zwischen dem Inkafürsten Zamore und dem gewalttätigen Spanier Don Gusman vorliegt, zu unspezifisch, und der einzige onomastische Bezug, der mehrfach erwähnte, aber nie auftretende Zamoro, wirkt allenfalls wie eine ironische Markierung. ${ }^{12} \mathrm{Da}$ ein fundierter literar- und motivgeschichtlicher Vergleich des Montezuma mit den Amerika-Dramen und -Opern des 17. und 18. Jahrhunderts noch aussteht, ist die Originalität von Friedrichs Bühnenwerk nicht abschließend geklärt. ${ }^{13}$

\section{Konzeption und Quellen}

Im Folgenden soll Friedrichs Heroisierung des fremden aztekischen Herrscherpaars untersucht werden. Legt die Stoffwahl eine spezifische Exotisierung nahe, ist die Heroisierung Montezumas und seiner Braut Eupaforice vorrangig durch die Gattung der Oper bedingt und somit in einem musik- und gattungsgeschichtlichen Kontext zu sehen. Wie wir aus der Korrespondenz mit seiner Schwester Wilhelmine und dem Grafen Francesco Algarotti wissen, zielte Friedrich, seitdem er im Spätsommer 1753 seine Tragedia per musica begonnen hatte, auf eine moderne mu-

11 Vgl. Ronald S. Ridgway: Voltairian bel canto: Operatic Adaptations of Voltaire's Tragedies. In: Studies on Voltaire and the Eighteenth Century 241, 1986, 125-154, besonders 138-143, sowie die ausgewogene Studie von Babette Kaiserkern: Spiegelungen: Friedrich II., Montezuma (1755) und Voltaire. In: Brunhilde Wehinger und Günther Lottes (Hg.): Friedrich der Große als Leser. Berlin 2012, 185-201.

12 Kaiserkern: Spiegelungen (Anm. 11), 200-201, sieht im sechs Mal genannten, aber „nie in persona auftretenden Zamoro“ eine „Chiffre des intertextuellen Epitextes“, in der Friedrich den Abgang Voltaires aus Berlin thematisiere. Zum Verhältnis zur Alzire vgl. auch die mehr essayistische als quellengestützte rezeptionshistorische Rekonstruktion der Opernerlebnisse des portugiesischen Gesandten, des Akademiepräsidenten Maupertuis und des Staatsministers von Podewils durch Georg Quander: Montezuma als Gegenbild des großen Friedrich oder: Die Empfindungen dreier Zeitgenossen beim Anblick der Oper ,Montezuma' von Friedrich dem Großen und Carl Heinrich Graun. In: Hellmut Kühn (Hg.): Preußen: Dein Spree-Athen. Beiträge zu Literatur, Theater und Musik in Berlin (Preußen: Versuch einer Bilanz. Ausstellungskatalog in fünf Bänden, Bd. 4). Reinbek 1981, 121-134, besonders 129-130. Gegen einen unmittelbaren Einfluss spricht auch, dass Friedrichs Montezuma-Tragödie eine frühere Episode der spanischen Eroberung Südamerikas behandelt, und die Religion bei ihm im Unterschied zu Voltaires Alzire nur eine nebensächliche Rolle spielt.

13 Vgl. Marion Fürst: Mit Federschmuck und Friedenspfeife. Der Amerikaner in der europäischen Oper des 17. und 18. Jahrhunderts. In: Musica 47, 1993, 21-24. Fürst vergleicht Friedrichs Montezuma ganz knapp mit Purcells Semi-Oper The Indian Queen und mit Jean Philippe Rameaus Ballet héroique Les Indes Galantes. Maehders zahlreiche Studien (Anm. 10) bleiben mehr additiv als komparativ. Motivgeschichtlich in eine breite Diachronie eingebettet ist die Studie von Susanne Detering: Friedrich II.: Camouflage und Selbstverteidigung im Bild Montezumas. In: Susanne Detering: Kolumbus, Cortés, Montezuma: Die Entdeckung und Eroberung Lateinamerikas als literarische Sujets in der Aufklärung und im 20. Jahrbundert. Weimar 1996, 196-214. 
sikdramatische Realisation: So bevorzugte er statt der traditionellen dreiteiligen $D a$ capo-Arien die knappere zweiteilige Arienform der gefühlvollen liedartigen Cavatine. ${ }^{14}$ Außerdem lockerte er die „strenge Scheidung von Handlung im Rezitativ und lyrischer Reflexion, Handlungsstillstand in der Arie" 15 auf und integrierte die Ballette an den Aktschlüssen in die Dramenhandlung. Vor allem aber löste er die Oper von dem gattungstypischen lieto fine, dem glücklichen Ende: ${ }^{16}$ Seine ,musikalische Tragödie ${ }^{6}$ endet mit dem Tod der indigenen Protagonisten Montezuma und Eupaforice. Dass Friedrich die Tradition der Opera seria aber nur modifizierte und nicht mit ihr brach, zeigt neben wenigen Da capo-Arien, die er den Hauptrollen Montezuma und Eupaforice zugestand, und der genretypischen Gefängnisszene, die zu Beginn des III. Akts die Peripetie einleitet, ${ }^{17}$ das exotische Dekor. Die „Auszierung der Schaubühne“, eine „neue Erfindung“ des königlichen Theaterdekorateurs Giuseppe Galli Bibiena, illustriert Exotik wie Gewalt der Handlung. So zeigt die Eingangsszene „drey große Gänge von Palmen in dem Kayserlichen Garten“, ein Gefängnis bildet das erste Bühnenbild des III. Aktes (Abb. 1), während die

14 Für seine Vorliebe führt Friedrich dramenökonomische Gründe an und beruft sich als Vorbild auf Hasse, vgl. den Brief Friedrichs an Wilhelmine, Markgräfin von Bayreuth: „Quant aux cavatines, j'en ai vue de Hasse qui sont infinement plus joilies qu les airs, et qui passent rapidement. Il ne faut de preprises que lorsque les chanteurs savent varier la musique; mais il me semble que, d'ailleurs, il y a de abus à répéter quatre fois la même chose" (Friedrich der Große und Wilhelmine von Baireuth, Bd. 2: Briefe der Königszeit 1740-1758, hg. von Gustav Berthold Volz, dt. von Friedrich Oppeln-Bronikowski. Leipzig 1926, besonders 266-270); zum Terminus ,Cavatine' vgl. Frauke Schmitz-Gropengießer: Cavata, cavatina/Kavatine, cabaletta. In: Handwörterbuch der musikalischen Terminologie, Lieferung 39, 2005. Vgl. Sabine Henze-Döhring: Friedrich der Große. Musiker und Monarch. München 2012, besonders 85-92, hier 86. Im italienischen Textbuch des Montezuma finden sich allerdings nicht jeweils zwei Da capo-Arien für die beiden Protagonisten, wie Friedrich im Brief an seine Schwester Wilhelmine vom 16. April 1754 noch vorsah, vielmehr nur eine einzige für Montezuma (I 1) und drei für Eupaforice (I 5, I 9 [Schluss I. Akt] und II 13 [Schluss II. Akt]), von denen zwei als effektvolle Aktschlüsse eingesetzt sind; vgl. Graun: Montezuma (Anm. 5).

15 Susanne Oschmann: Gedankenspiele: Der Opernheld Friedrichs II. in Preußen. In: Klaus Hortschansky (Hg.): Opernheld und Opernheldin im 18. Jabrhundert. Aspekte der Librettoforschung. Ein Tagungsbericht (Schriften zur Musikwissenschaft aus Münster; 1). Hamburg und Eisenach 1991, 175-193, hier 189. Auf den Montezuma geht Oschmann in ihrem Überblick aber nur knapp ein (184-187).

16 „Ab dem Jahr 1750 endeten beinahe alle in Berlin aufgeführten Werke - mit Ausnahme der Hochzeits- bzw. Sommeropern - ohne lieto fine. Für die Opern Coriolano, Il Mithridate, Britannico, Semiramide, Montezuma, I Fratelli Nemici und Merope wählte man statt der zeitgenössischen Gattungsbezeichnung Dramma per musica jeweils den Untertitel Tragedia per musica" (Claudia Terne: Friedrich II. von Preußen und die Hofoper. In: Michael Kaiser und Jürgen Luh (Hg.): Friedrich der Große und der Hof. Beiträge des zweiten Colloquiums in der Reibe „Friedrich300“vom 10./11. Oktober 2008, 1-38, hier 29, http://www.perspectivia.net/ publikationen/friedrich300-colloquien/friedrich-hof/Terne_Hofoper, 29. März 2016).

17 Siehe Michele Calella: Die Opera seria im späten 18. Jahrhundert. In: Herbert Schneider und Reinhard Wiesend (Hg.): Die Oper im 18. Jabrhundert. Handbuch der musikalischen Gattungen, Bd. 12. Laaber 2001, 45-62, hier 53. Vgl. dazu den informativen Überblick mit einigen Hinweisen zur musikalischen Gestaltung solcher Szenen von Helga Lühning: Florestans Kerker im Rampenlicht. Zur Tradition des Sotterraneo. In: Helga Lühning und Sieghard Brandenburg (Hg.): Beethoven zwischen Revolution und Restauration. Bonn 1989, 137-204. 


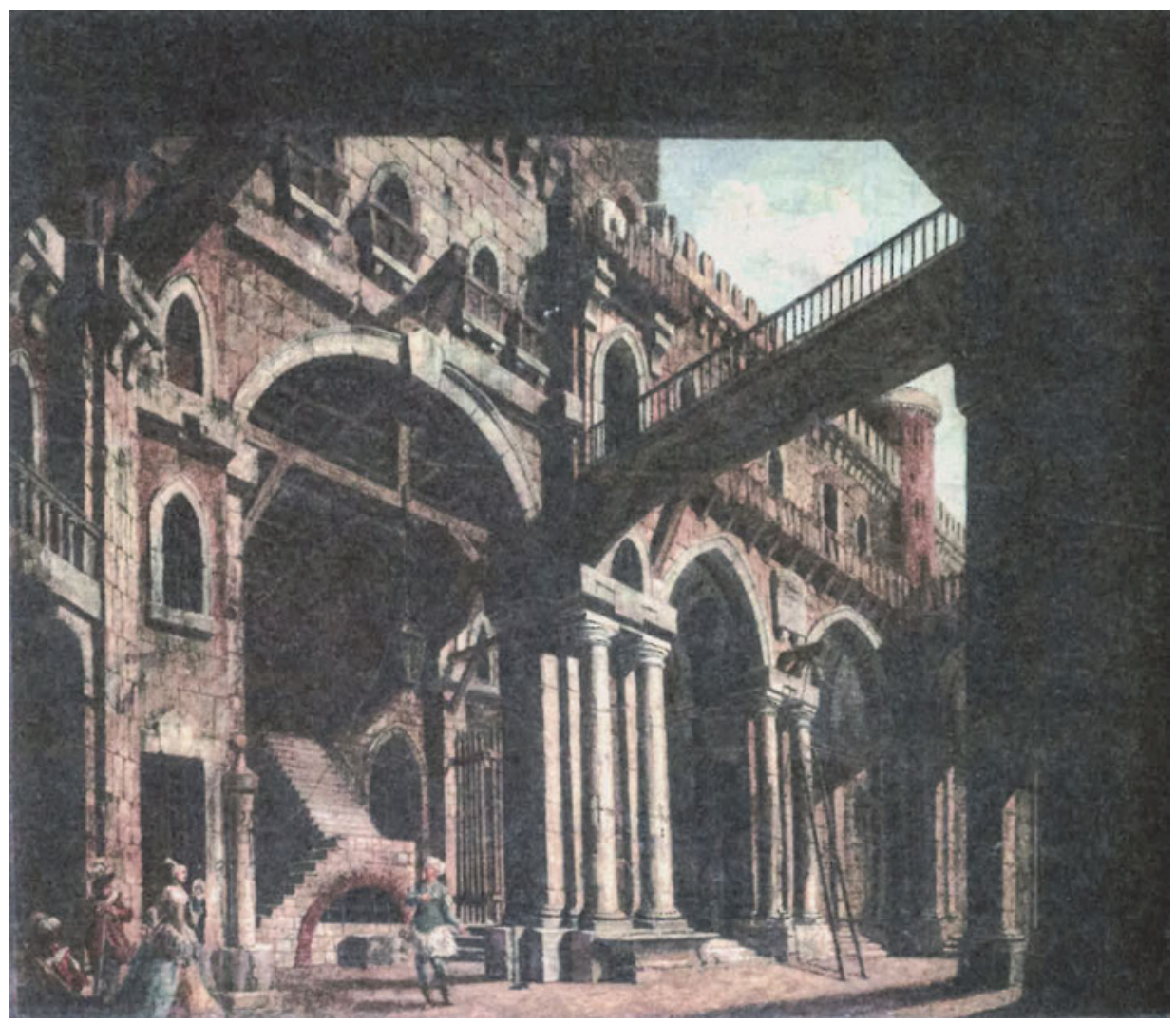

Abb. 1: Carl Friedrich Fechhelm: Bühnenbild zu Montezuma, III 1, 1755, Öl auf Leinwand, $90 \times 100 \mathrm{~cm}$, Potsdam, Stiftung Preußische Schlösser und Gärten Berlin-Brandenburg, GK I 12008 .

Schlussszene „ein[en] große[n] mit Säulen umgebene[n] Hof“ zeigt, „zwischen welchen man einen Theil von der in Brand gesteckten Stadt Mexico siehet“ (A4r). Ein überliefertes Kostümbild bestätigt die äußerliche Exotisierung des Personals (Abb. 2 und 3).

Inwieweit bestimmt eine Heroisierung des Aztekenherrschers Friedrichs Tragödie, welche die militärische Niederlage dramatisiert, die Motecuzoma II. (,Der Traurige') im Jahre 1519 gegen die spanischen Eroberer um Hernán Cortés erlitt? Eine heroisierende Wirkungsabsicht liegt durchaus nahe, zumal Friedrich seine antispanische Opera seria ausdrücklich als aufklärerisches Medium erachtete, „à reformer les mœurs et à detruire les superstitions" ${ }^{\text {" }}{ }^{18}$ Heroisierungen kommen auf der

18 Zur Helden- und Herrscherdarstellung unter anderem Reinhard Wiesend: Der Held als Rolle: Metastasios Alexander. In: Klaus Hortschansky (Hg.): Opernheld und Opernheldin im 18. Jabrhundert. Aspekte der Librettoforschung. Ein Tagungsbericht. Hamburg und Eisenach 1991, 139-152. Vgl. Brief Friedrichs II. an Francesco Algarotti vom Oktober 1753. In: Francesco Algarotti: Briefwechsel mit Friedrich II., hg. von Wieland Giebel, nach dem ital. 


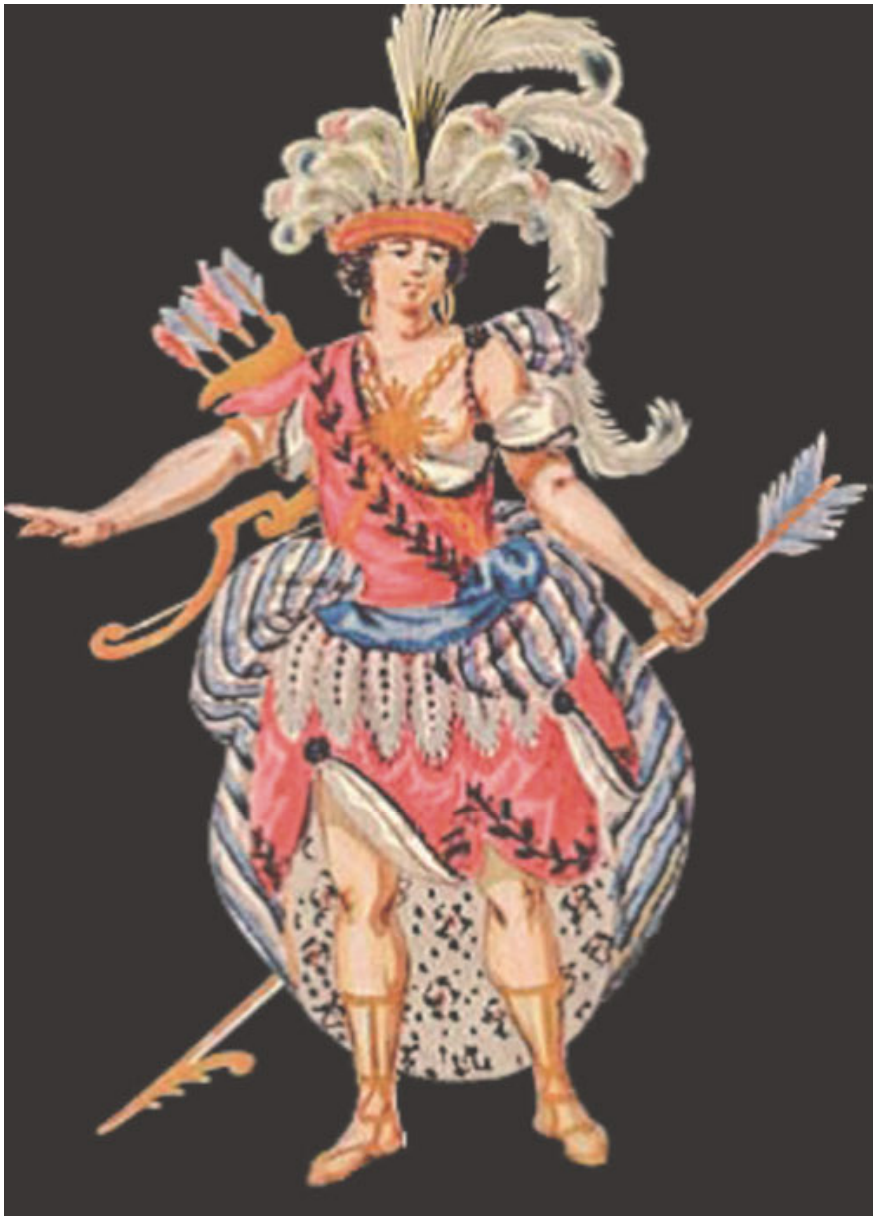

Abb. 2: Carl Friedrich Fechhelm: Re Americano, Figurine für den mexikanischen Kaiser Montezuma, 1754, Berlin, Sammlung Louis Schneider.

Bühne in Konfiguration, Handlung und Sprache zum Ausdruck. Sie setzen innerhalb des Figurenpersonals neben einer moralisch vorbildlichen Gestalt ein Kollektiv voraus, das den Helden verehrt, und einen Gegenspieler, der ihn - ex negativo kontrastiv verherrlicht. Da die oft typologisch markierte heroische Konfiguration erst in der Inszenierung ihre performative Dynamik gewinnt und durch die Handlung wie sprachliche Kommunikation beglaubigt wird, ergibt sich ein gezieltes close reading als Vorgehen: $\mathrm{Ob}$ und wie Montezuma und seine Braut in Friedrichs Tragödie heroisiert werden, wird zunächst auf der Handlungsebene untersucht und durch die Analyse der Diskursebene perspektiviert.

Original aus dem Jahr 1799 mit einem Vorwort des Übersetzers Friedrich Fursten. Berlin 2008, 112-114. 


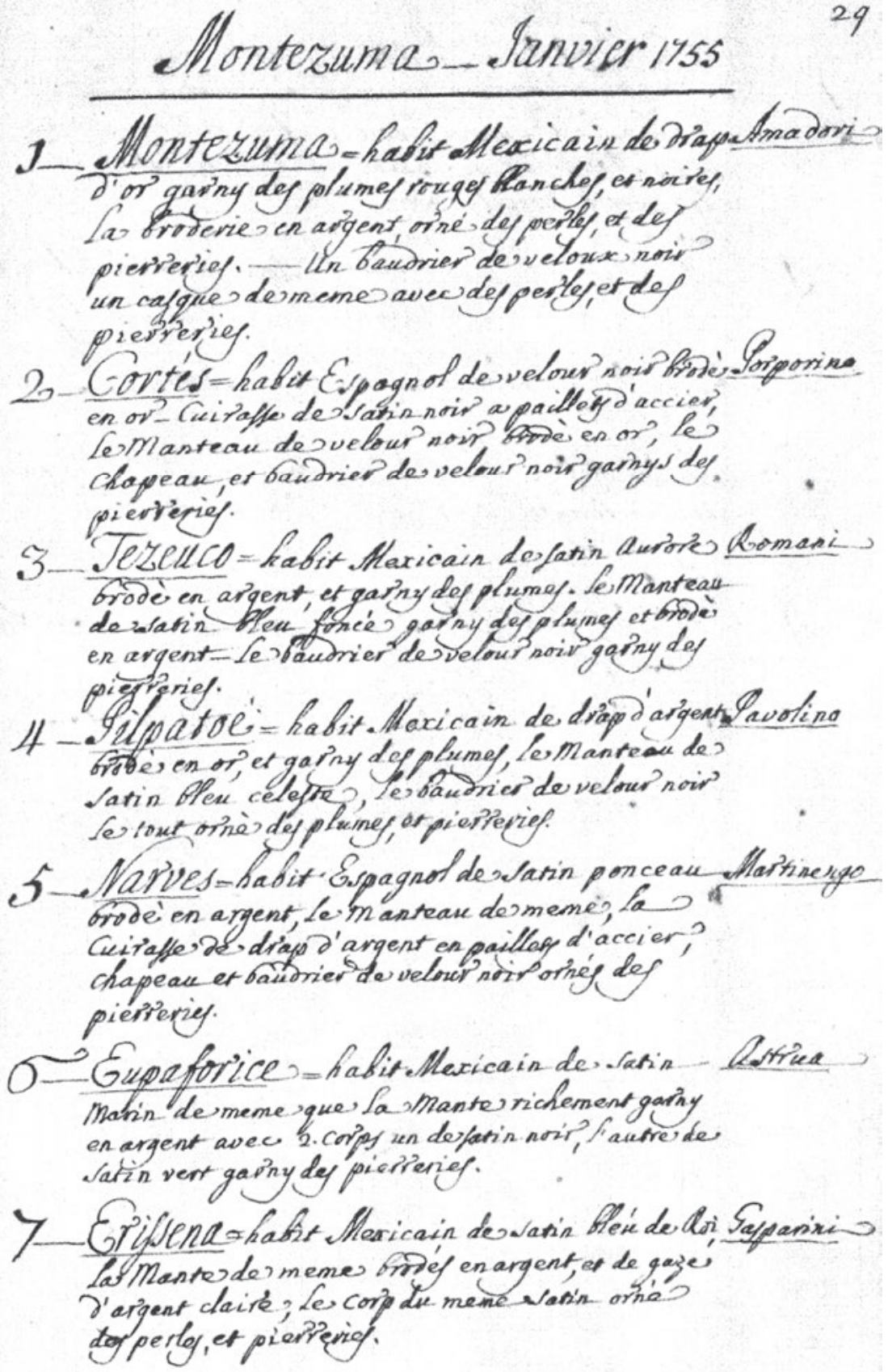

Abb. 3: Kostüm-Inventar der Oper Montezuma, Berlin, Geheimes Staatsarchiv Preußischer Kulturbesitz, I. HA Geheimer Rat, Rep. 36 Hof- und Güterverwaltung, Nr. 2627. 
Für seine Tragödie hat Friedrich zwar historiografische Werke konsultiert, vor allem die maßgebliche Abhandlung von Antonio de Solís y Ribadeneyra, ${ }^{19}$ aber die komplexe Geschichte der Eroberung Mexikos stark gerafft und die innerspanische Auseinandersetzung zwischen Cortés und Narves ganz übergangen. Auch wenn die kriegerische Kulturkollision zwischen den Europäern und den indianischen Ureinwohnern relativ spät ein literarisches Sujet geworden war, war Friedrichs Montezuma keineswegs die erste musikdramatische Gestaltung der spanischen Eroberung des Aztekenreichs. Vorausgegangen war ihm Antonio Vivaldis Motezuma aus dem Jahre 1733 nach einem Libretto von Girolamo Giusti. ${ }^{20}$ Giusti/Vivaldi hatten freilich den Stoff operngemäß verharmlost, indem der Krieg zwischen der mexikanischen und spanischen Partei durch die kultur-, religions- und parteiübergreifende Liebe zwischen Motezumas Tochter Teutile und Fernandos Bruder Ramiro befriedet wird. Das Opfer eines Menschenpaars, welches das von den Mexikanern befragte Orakel verlangt, gilt schließlich mit dem christlichen Sakrament der Ehe als erfüllt. Friedrichs Montezuma kommt der historischen Wahrheit viel näher, indem er auf ein glückliches Ende verzichtet und stattdessen den Tod des Aztekenherrschers und das grausame Gemetzel darstellt, das die Spanier unter den Azteken anrichteten.

Doch mehr als historisches Interesse prägt aufklärerische Parteinahme Friedrichs Tragedia per musica. In eindeutiger Sympathielenkung wird Montezuma als großmütiger Fürst vorgestellt, der gerade wegen seines Seelenadels Opfer der Grausamkeit und Habgier der spanischen Eroberer wird. Es ist bezeichnend, dass in der tragischen Konstellation der Gegenspieler und „Tyrann" ${ }^{21}$ Cortes nicht namentlich genannt wird, sondern im Fremdkollektiv der „Spanier“ oder „barbarischen Gäste" aufgeht:

Der damahls regierende Kayser von Mexico, Montezuma, erlaubte den Spaniern auf guten Glauben, den Eintritt in sein Reich; aber, er spürte hernach allzuspät die Wirckung eines gar zu leichtgläubigen Vertrauens, und einer unzeitigen Großmuth, als die ihm das Leben kostete. Das betrübte Ende dieses guten Monarchen, welcher der Grausamkeit und dem Geitze seiner Gäste barbarischer Weise aufgeopfert wurde, hat also den Stoff zu diesem Trauerspiele gegeben. $\left(\mathrm{A} 3^{\mathrm{r}}\right)$

Noch schärfer konturiert Tagliazucchis dramentheoretische Leservorrede zur französischen Prosatragödie die Opposition der Konstellation:

On trouvera danz Montézuma le Heros qu'exige la Tragedie, un tendre Pére de son Peuple dont il fait les delices, un Prince juste, sage, magnanime, modeste dans sa prospérité, intrepide dans ses malheurs: S'il est sensible à une tendre passion, son amour n'est que

19 Die französische Übersetzung von 1704 war in drei der acht friderizianischen Bibliotheken vorhanden, vgl. Krieger: Friedrich der Große und seine Bücher (Anm. 1), 125. Zu den Differenzen und Übereinstimmungen der Montezuma-Figur bei Antonio de Solís vgl. Kaiserkern: Spiegelungen (Anm. 11), 197-199.

20 Während Giustis Libretto überliefert ist, galt die Partitur der Oper lange als verschollen, um deren imaginäre Aufführung der kubanische Schriftsteller Alejo Carpentier seinen Roman Concierto baroco (1974) kreisen lässt.

21 So Friedrich in einem Brief an Algarotti vom Oktober 1753 (Anm. 18). 
l'effet de sa vertu: s'il pousse trop loin la confiance et la sécurité, c'est un deffaut naturel aux grandes Ames, et qui semble leur être permis. Cortés est fourbe, avare, et cruel; ces caractéres opposés produisent sur le Theâtre les plus grands éffets: ils y sont ce l'ombre est dans le Tableau. ${ }^{22}$

Montezuma wird als tragischer Held präsentiert: Seine Eigenschaften „Vater seines Volks“, „gerecht“, „großmütig“, „bescheiden im Reichtum, unerschrocken im Unglück“ typisieren ihn als guten Fürsten, seine Schwächen, „vertrauensselig“ und „sorglos“, werden als Zeichen seiner Seelengröße aufgewertet. Sein Gegenspieler Cortes wird dagegen nur knapp als „schlau“, „habgierig“ und ,grausam“ charakterisiert und explizit als Kontrastfigur bezeichnet. Die beiden feindlichen Lager sind zwar nach der Zahl der Statisten und der Ballette ausgewogen;23 doch hinsichtlich der stimmlichen Präsenz dominiert eindeutig die mexikanische Partei, auf die fünf der sieben Gesangsrollen entfallen. Im Zentrum stehen Montezuma, der „Kayser von Mexico“, und seine königliche Braut Eupaforice. Dem Liebespaar ist jeweils eine Dienerfigur zugeordnet, ${ }^{24}$ hinzu tritt der kaiserliche General Pilpatoe. Dagegen repräsentieren nur Ferdinando Cortes als „Haupt der Spanier“ und der Hauptmann Narves die spanische Partei..$^{25}$

Diese Parteinahme setzt sich in der Anlage der dreiaktigen Tragödie fort. Der I. Akt gehört ausschließlich den Mexikanern, im II. und III. Akt kommt es auf der Bühne zu den solchermaßen perspektivierten Kulturkollisionen.

22 Vgl. Tagliazucchi: Au Lecteur. In: Anon. [Friedrich II., Kg. von Preußen]: Montezuma. Tragédie en trois actes (mit frz. Vorrede von [Giovan Pietro] Tagliazucchi). Berlin (J. G. Michaelis) o. J. [1755], 3-7, hier 4, Übersetzung: ,Man wird in Montezuma den Helden finden, den die Tragödie verlangt, einen zärtlichen Vater seines Volkes, dessen ganze Freude er ist, einen gerechten Herrscher, weise, großherzig, bescheiden in seinem Reichtum, unerschrocken im Unglück: Wenn er für eine zärtliche Leidenschaft empfänglich ist, dann ist seine Liebe nichts Anderes als das Ergebnis seiner Tugend: wenn er zu vertrauensselig ist und sich zu sicher fühlt, dann ist das ein natürlicher Fehler großer Seelen, der ihnen erlaubt zu sein scheint. Cortes ist hinterlistig, habgierig und grausam; diese gegensätzlichen Charaktere sind auf der Bühne am effektvollsten. Sie haben denselben Effekt wie der Schatten auf einem Bild.‘

23 So entsprechen sich die Gefolgsleute von Montezuma und Cortes. Die je sechs Tänzerinnen und Tänzer agieren einmal als spanische Matrosen, dann als „Mexicaner“, um sich schließlich in der tragischen Schlussszene, welche die Plünderung Mexikos durch die Spanier darstellt, zu mischen.Vgl. die letzte Szenenanweisung: „Die Spanier laufen zur Plünderung. Die Täntzer mischen sich in spanischer Kleidung unter die Soldaten, plündern die Stadt, und bemächtigen sich der Mexicanischen Frauen, die sich vertheidigen, und sehr lebhafft ein Ballet formiren, welches sich mit dem Chor anhebet, so das Mexicanische Volck singet, das erschrocken und in Verwirrung die Flucht nimmt." (III 5, 139)

24 Tezeuco, ein „Bedienter der Kayserlichen Krone“, sowie Erixene, „Vertraute der Königin“.

25 Während Montezuma, den in der Berliner Uraufführung der berühmte Alt-Kastrat Giovanni Amidori spielte, und seine Braut Eupaforice, verkörpert von der damaligen Starsopranistin Giovanna Astrua, jeweils vier Arien singen, entfallen auf Cortes, verkörpert durch den Alt-Kastraten Porporino, lediglich drei Arien. 


\section{Handlungsstruktur}

Der I. Akt stellt den mexikanischen Hof als ein Idyll des Friedens vor. Doch bleibt das exotische Setting äußerlich, denn der Diener Tezeuco bezeichnet seinen Herrn Montezuma wie einen aufgeklärten europäischen Fürsten als „Tröster“, „Wohltäter“, „Erretter“, und „Vater deines Volcks“ - im Italienischen bezeichnenderweise „Padre della Patria“ (I 1, 14-15), was dieser - wie Friedrich in seinem AntiMachiavel - als seine bloße „Pflicht“ („,il mio dovere“) abtut (I 1, 14-15). Eingespiegelt wird die ,fremde Wahrnehmung' aber in der Schilderung der spanischen Conquistadores. Der Bericht des kaiserlichen Generals Pilpatoe invertiert die übliche europäische Alteritätsperspektive auf die Neue Welt. Den Azteken erscheinen die Europäer nun als „die fremden und furchtbahren Helden“, die „auf schwimmenden | Und starcken Festungen große Meere durchschiffe[n]" (I 2, 17), ihre Feuerwaffen seien „Ungeheuer“, so „schnell [...], daß kein Gedancke es begreifen kann“ (I 2,19) und man nicht wisse, ob die dreihundert „Fremdlinge“, „Menschen oder Götter sind“ („vomini, o Die“) (I 2, 20-21). Trotz der Warnungen seiner besorgten Geliebten und seines Generals gewährt Montezuma den stolzen Fremden Gastrecht, die durch ihren Hauptmann Narves einen gebührenden Empfang für „die Helden“ fordern, „welche der Himmel mit dem Donner hat bewafnen wollen“ (I 7, 39). Den I. Akt beschließt eine Kontrastmontage: Eupaforice, „zwischen Hoffnung und Furcht" hin- und hergerissen, erklärt sich in einer märtyrerhaften Gebets- und Liebesarie zum Liebestod für Montezuma bereit:

Höret meine Wünsche, o ihr Götter!

Verlanget ihr ein Opfer?

Tödtet mich, hier ist meine Brust,

Aber rettet meinen Geliebten! (I 9, 47)

Dieser ängstlichen Arie folgt ein „Ballet von Schifs-Leuten“, das die selbstherrlichen spanischen Konquistadoren präsentiert.

Montezuma, so kann festgehalten werden, hat in seinem Hof das kollektive verehrende Gegenüber, das jedoch seine Entscheidung, den Fremden Gastrecht zu gewähren, anzweifelt. Ein mögliches Heldentum Eupaforices deutet sich bereits in ihrer Arie an und konkretisiert sich mit dem eskalierenden Konflikt.

Im II. Akt gewinnt die Kulturkollision Gestalt: Cortes tritt erstmals auf, und die spanische Partei wird isoliert vorgestellt. Im Dialog mit seinem draufgängerischen Hauptmann Narves erweist sich Cortes als perfekter Macchiavellist, wie ihn Friedrich II. in seinem Anti-Machiavel geschildert hatte:

Die Eroberung von Mexico soll meiner Klugheit

Allein gehören. Wir müssen uns der List

Und des Betrugs bedienen; ich will,

Daß Montezuma durch sich selbst falle, und sich

Eines Verbrechens schuldig mache, damit ich

Das Recht haben möge, ihn zu bestraffen.

Laßt uns noch Verstellung gebrauchen. (II 1, 49, 51) 
Die italienische Wendung „Dissimuliam“ („Dissimulons encore“ [frz. 26]), für ,verstellen', die Cortes am Ende des Dialogs mit Narves als Maßregel für das Verhalten gegenüber Montezuma ausgibt, verrät die doppelte Zielrichtung des Angriffs. Denn die dissimulatio galt als jesuitische Verhaltensregel, seitdem Machiavellis Maxime der simulatio diskreditiert war. Mit der Geringschätzung der „gottlosen und verfluchten Heyden“ (II 1, 51) kontrastiert die gastfreundliche Begrüßung der „stranieri Eroi“ durch das mexikanische Volk in Gestalt des Chors:

Venite, intrepidi stranieri Eroi

Tolti al terribile furor del mare.

Cari, e pregevoli sarete a noi,

E al nostro amabile Imperator. ${ }^{26}$

Montezumas Großmut, Cortes zu seiner Hochzeit einzuladen und die spanischen Truppen in den kaiserlichen Palast einzulassen, kontrastiert mit der Heimtücke des Cortes, der mit seinen Leuten „die Kayserliche Leib-Wacht“ überwältigt und die Maske der Verstellung fallen lässt: Er will nicht nur Montezuma gefangen nehmen, sondern „sogar seine Braut für [s]ich rauben“ (II 5, 65). Den Heiratsplan erklärt Cortes dem erstaunten Hauptmann Narves (,Wie? Könntest du eine Barbarin heyrathen! Eine Heydin! O Himmel“ [II 5, 67]) mit machiavellistischem Machtkalkül:

Ja, Freund, man muß alles unserem

Nutzen aufopfern. Verstellung, Kühnheit,

So gar die Liebe,

Alles muß dienen, sich einer

Eroberung zu versichern [...]. (II 5, 67)

Erst in dem Streitgespräch mit Montezuma, welches das Zentrum der Tragödie bildet, camoufliert Cortes den machtpolitischen Staatsstreich als christlichreligiöse Mission: „Unser Gesetz will, daß wir die Götzen-Diener verabscheuen, | Welche barbarischen Göttern menschliche Opfer bringen“" (II 7, 71). Als Montezuma sich gegen seine Entmachtung mit dem Degen zur Wehr setzt, wirft ihm dies Cortes zynisch als Bruch der "Gast-Freyheit“ vor und lässt ihn unter dem Vorwand der eigenen Sicherheit in Ketten legen. Doch Montezuma fügt sich nicht in die Rolle des unterwürfigen Entmachteten. Indem er Cortes die Rolle eines Tyrannen zuweist, stilisiert er sich selbst in der Schlussarie des siebten Auftritts zum todesmutigen Märtyrer:

Ich empfinde alle Schrecken meines tyrannischen

Schicksals; ich werde mich aber nicht

Durch Kleinmuth von der Furcht

Ueberwältigen lassen, und du sollst

Mich nicht zittern sehen.

26 „Kommt ihr tapfere fremde Helden, die ihr | Von der schrecklichen Wuth des Meeres gerettet worden. | Ihr sollt uns und unserm | Geliebten Kayser werth und willkommen seyn." (II $2,53)$. 
Barbar! Wo ist der Tod?

Komm, und nimm mir das Leben.

Es ist besser tapfer umkommen,

Als unter Scham und Spott

Das Leben zu erhalten. (II 7, 79)

Nachdem Eupaforice vergeblich die Freilassung Montezumas erbeten und sich in einer Rührszene von dem Gefangenen verabschiedet hat, macht Cortes ihr einen Heiratsantrag. Sein kolonialistisch-persuasives Argument, sie gehöre kulturell eher zu Europa als zu Mexiko, verfängt aber nicht:

CORTES. [...]

Die Annehmlichkeiten

Die dir die Natur verliehen hat,

Gehören nicht für einen barbarischen Printzen, du könntest deine Größe erhalten,

Wenn - - - Ach glaube mir,

$\mathrm{Daß}$ die Europäer mehr als die Mexicaner

Deinen Werth zu schätzen wissen, und daß es Hertzen giebet,

Die dich besser zu verehren wüsten.

EUPAFORICE. Ich verabscheue die Europäer, wenn ihre

Sitten den deinigen gleichen. (II 9, 87)

Auf die erpresserische Drohung, nur ihre Einwilligung könne den gefangenen Montezuma retten, reagiert Eupaforice in einer ambivalenten Arie zunächst mit märtyrerhafter Todesbereitschaft, dann mit einem Fluch:

Barbar, dessen vermessener Anblick (Zum Cortes.)

Mir ein Abscheu ist, du willst mir

von Liebe reden?

$[\ldots]$

Nein Unwürdiger, du sollst niemahls

Die Frucht deiner Verbrechen genießen,

Du bist ein grausames Ungeheuer!

Ich schwöre, daß ich meinem Geliebten

Bis zum Tode treu seyn will.

Möchte doch heute auf dich

Und auf deine gottlosen Gefährten

Der Zorn des Himmels seinen

Blitz fallen lassen! (II 9, 89, 91)

Mit der Peripetie, der Gefangennahme Montezumas, steigt Eupaforice zur heroischen Gegenspielerin von Cortes auf. Sie organisiert unter der Devise „Entweder überwinden, oder sterben“ (II 11, 95) eine militärisch-politische Gegenoffensive und schwört trotz ungünstiger Nachrichten mit einer heroischen Rede ihre engsten Vertrauten auf den Kampf gegen die Spanier ein. ${ }^{27}$

27, „...] Ich selbst will an der Spitze seyn, | wir wollen unsere Tyrannen | ausrotten“ (II 12, 99). Für „diese[n] große[n] Gedancke[n]“ (ebd.), „entweder unsern Monarchen | retten, oder mit ihm sterben“ (ebd.), erntet sie Zustimmung, so dass den II. Akt ein „Ballet von Mexicanern“ hoffnungsfroh beschließt (103). 
Den III. Akt eröffnet eine für die italienische Opera seria genretypische Gefängnisszene:28 In einem langen Accompagnato-Monolog räsonniert der gefangene Montezuma über den jähen Glückswechsel und mahnt sich zu stoischer Geduld, bis er vom - ebenfalls genretypischen - Besuch seiner Braut Eupaforice überrascht wird. Auch wenn sie ihm mitteilen muss, dass auf keine militärische Unterstützung von außen mehr zu hoffen sei, finden beide Trost in ihrer unverbrüchlichen Liebe. In rasch wechselnder, gelegentlich gar stichomythischer Rede, die in zwei - für die Berliner Oper ganz untypischen - Duetten gipfelt, den einzigen in Montezuma, versichern sich die beiden ihrer Bereitschaft zum Liebestod:

EUPAFORICE. Ach! Mit einer so reinen Liebe

MONTEZUMA. Ach! Mit einer so schönen Treue

BEYDE. Müßen wir entweder herrschen,

Oder zusammen sterben. (III 1, 115)

Graun hat die Kerkerszene als Peripetie musikalisch markiert, indem er sie wie die Katastrophe am Schluss mit einem umfangreichen Accompagnato-Rezitativ beginnen lässt - die Streicher, die mit den Achtel- und Sechzehntel-Akkordbrechungen einen Klangteppich weben, bekräftigen die resignativen Gedanken und Emotionen, die Montezuma im Monolog äußert. Graun weicht in der Kerkerszene von der üblichen Abfolge Rezitativ - Arie ab und fügt vom Accompagnato über SeccoPartien und die eingelegte Kavatine eine große Szene bis zum Duett der Liebenden zusammen (Abb. 4). Auf diese Weise integriert er Eupaforice in die Heroisierung Montezumas.

Da Cortes von Eupaforices Geheimplan, die Spanier im Schlaf zu überwältigen, erfährt und seinerseits nun die Mexicaner, die vom verzweifelt kämpfenden Pilpatoe angeführt werden, umbringen lassen will, entschließt sich das Liebespaar zur gemeinsamen Flucht. Doch während Cortes den mexikanischen General Pilpatoe gefangen nimmt und ihm „die grösten Martern“ androht (III 4, 125), wird das flüchtige Kaiserpaar von Narves überwältigt. Cortes wirft Montezuma in einem affektisch aufgeladenen Streitdialog Hochverrat vor: „Du bist der einzige Urheber der Rebellion [...], | Du hast die gantze Zusammenverschwörung angesponnen“ (III 5, 129), und eröffnet ihm das Todesurteil: „Wisse also, | Daß die Todes-Strafe dich erwartet“ (ebd.). Während Montezuma sein Verhalten als Notwehr rechtfertigt, verteidigt Eupaforice ihren Widerstand mit naturrechtlichen Argumenten: „Bedencke, daß keine Creatur auf der Welt lebet, | Welche sich unterdrücken ließe, ohne sich zu wehren, | Und daß wir dasjenige gethan haben, | Was unsere Pflicht von uns erforderte" (III 5, 131). Cortes' Angebot, Montezuma am Leben zu lassen, wenn dieser auf Eupaforice verzichten würde, lehnen beide ab, und Eupaforice unterscheidet in einer Arie den militärischen Sieger Cortes von den „wahren Helden“, die „menschliche Empfindungen“ haben (III 5, 132-133). Während Montezuma und seine Getreuen „zum Gericht geschleppt“ werden, gehört der

28 Siehe Calella: Die Opera seria im späten 18. Jahrhundert (Anm. 17), 53. 

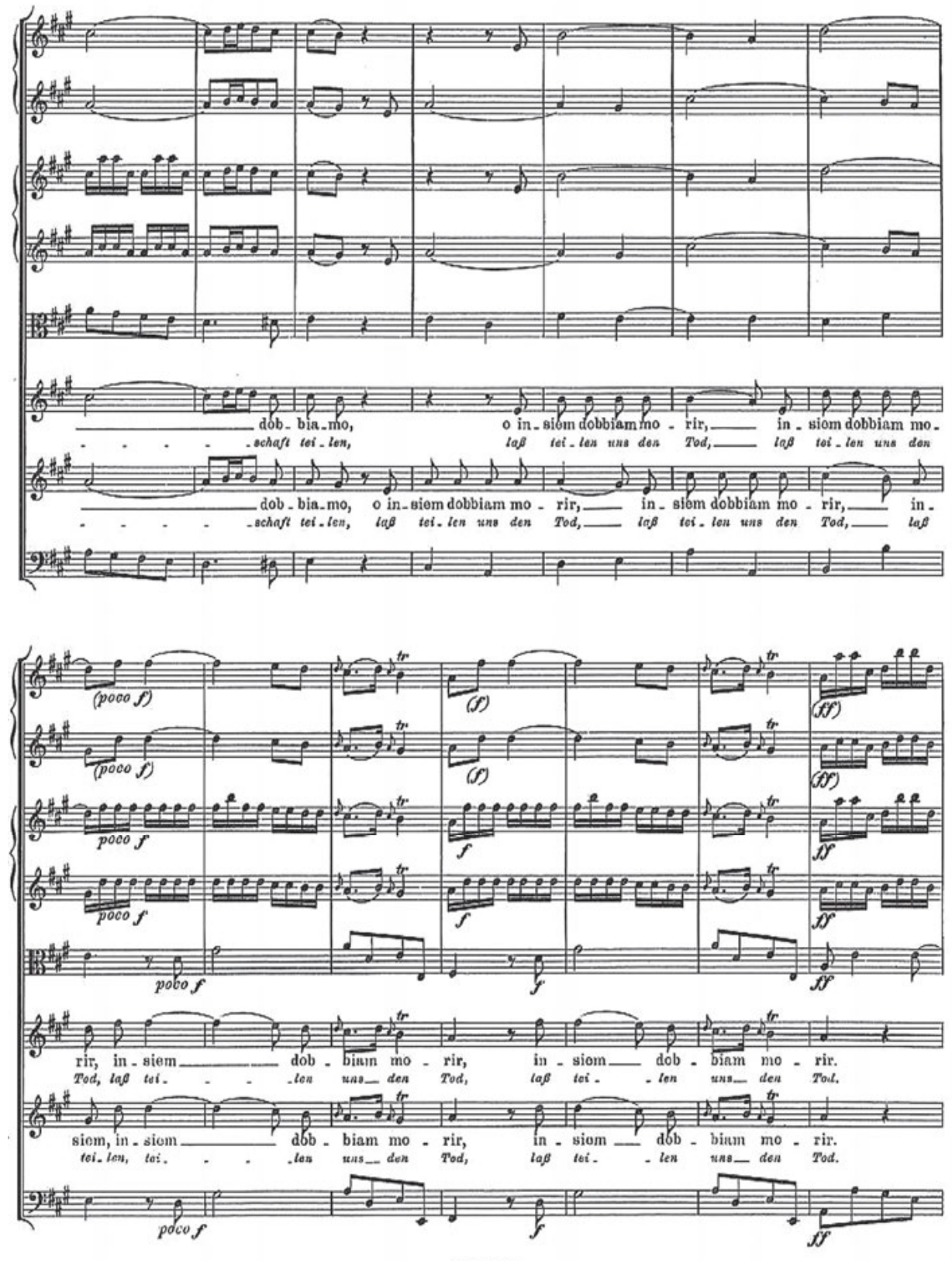

D. D.T. XY.

Abb. 4: Auszug aus dem Duett von Montezuma und Eupaforice in III 1. 
Schluss Eupaforice, die sich als „eine rächende“ und Cortes „verfolgende Furie“ gebärdet: Sie hat die Stadt in Brand setzen lassen, um den Spaniern weder Untertanen noch Reichtümer ausliefern zu müssen und tötet sich schließlich in einem heroischen Akt selbst - „Sie stößt sich einen Dolch in die Brust", um sich „nicht von | Meinem zärtlichen Liebhaber trennen“ zu müssen: „Unsere Seelen sollen sich in diesem Augenblick vereinigen“ (III 5, 137, 139). Diese Schlussszene mit Eupaforices Accompagnato (213) wächst direkt aus der letzten Arie heraus und vermittelt so musikalisch die Katastrophe.

Den Schein des Missionars im Dienste der spanischen Majestät, den Cortes noch mühsam wahrt, als er den Befehl zu Plünderung und Tötung der Einwohner gibt, widerlegt die grausame stumme Szene, die zum Chor der besiegten Mexikaner überleitet, als bloßen Vorwand:

Die Spanier laufen zur Plünderung. Die Täntzer mischen sich in Spanischer Kleidung unter die Soldaten, plündern die Stadt, und bemächtigen sich der Mexicanischen Frauen, die sich vertheidigen, und sehr lebhafft ein Ballet formiren, welches sich mit dem Chor anhebet, so das Mexicanische Volck singet, das erschrocken und in Verwirrung die Flucht nimmt. (III 5, 139)

Der Chor, der das Stück beschließt, ruft die „gerechten Götter“ um Hilfe: „Errettet uns! | Laßt euch zum Mitleid bewegen!“ (III 5, 141).

\section{Heroisierungen und Deheroisierungen}

Im I. Akt erscheint Montezuma vor allem dadurch als heroische Figur, dass sein Hof ihn loyal verehrt. Montezumas Gefangennahme erzeugt ein heroisches Vakuum, das Eupaforice sukzessive füllt. Indem Montezumas Braut das Heft des Handelns übernimmt, wird sie wie dieser zur heroischen Figur. Die Heroisierung der beiden Figuren beschränkt sich nicht nur auf die Handlungsebene, sondern erfolgt ebenso auf der musikalischen und sprachlich-diskursiven Ebene.

\subsection{Perspektivismus von Heroisierung und Deheroisierung}

In einem Drama mit Held und Gegenspieler sind Heroisierung und Deheroisierung wechselseitig gebrochen. Dieser Perspektivismus zeigt sich in Friedrichs Montezuma ebenso wie eine dramatische Dynamik in den Selbst- und Fremdcharakterisierungen. Während die mexikanische Partei die Eindringlinge zunächst als „Fremde“, „Helden“, „Spanier“, ja sogar „Götter“ bezeichnet, gebrauchen die Spanier von Anfang an den Begriff ,Barbar", um die indigene Gruppe zu diskreditieren: ,Barbar' (,Barbaro' / ,Barbari') fungiert hier als Antonym zu ,Held' (,Eroe' / ,Eroi'). So eröffnet Narves den ersten innerspanischen Dialog mit dem Satz: „Hier sind wir endlich, dem Himmel sey Danck! | In den Mauern, wo der Barbar seinen Hof hat" (II 1, 49) und diskreditiert damit später den Heiratsplan von Cortes (II 5, 67). Cortes benutzt den Begriff relativ selten: Zwar nennt er einmal den Diener Tezeuco einen „Barbare[n]“ (II 5, 65), den kaiserlichen General Pilpatoe bezeich- 
net er verächtlich als „Mexicaner“ („Soldaten, jagt diesen Mexicaner| Aus dem Pallast fort" [II 7, 73]), doch verwendet er hauptsächlich strafrechtliche Termini. So beschuldigt er den Aztekengeneral der „Untreue“ („Du bist ein Rebelle“ [III 4, 125]) und wirft dem auf der Flucht ergriffenen Montezuma Verfassungsbruch vor: „Du bewaffnest das rebellische Reich, | Du stifftest Meutereyen, du suchst | Mein Blut zu vergießen“(III 5, 127).

Unter den Azteken dagegen signalisiert die Verwendung des Begriffs ,Barbar die Anagnorisis, den tragödienspezifischen Umschlag von Unkenntnis in Kenntnis: Montezuma bezichtigt Cortes, nachdem er dessen Falschspiel durchschaut hat, als „Barbar[en]“ und „barbarische[n] Feind“ (II 6, 69, 73), und dämonisiert ihn gar zum „abscheuliche[n] Ungeheuer [...], | Welches die Hölle ausgespien hat" (II 7, 75). Auch Eupaforice schimpft Cortes gleich zwei Mal einen „Barbar[en]", als er sie zur Heirat überreden will (II 9, 89). Mit der Peripetie kommt es zur Opposition einer ethnisch bestimmten kollektiven Identität und Alterität: Im antonomastischen Ethnikum „Mexicaner“ grenzt man sich von den „Spanier[n]“ ab (II 11, 95), die man überdies moralisch als „Tyrannen“ pejorisiert (II 12, 99). Als Montezumas Fluchtversuch zum Vorwand für einen Hochverratsprozess dienen soll, verweigert er den juristischen Diskurs, auf den sich Cortes verlegt, und kriminalisiert seinerseits das Verhalten von Cortes: „Barbarischer Räuber meines Reichs, und du unterstehst dich noch | Mich einen Rebellen zu nennen?" (III 5, 127). Er beschuldigt ihn einer falschen Propaganda („da alle Sachen | Einen andern Nahmen bekommen“ [III 5, 129]), nennt ihn „Tyrannen“ (ebd.) und reklamiert Religionsfreiheit (ebd.). In den Vorwurf, dem angeblichen Verfechter des ,richtigen' Glaubens jegliche Religion abzusprechen, stimmt auch Eupaforice ein, für die Cortes ein „Gottloser! Unmenschlicher!“ ist (III 5, 131), dessen „Barbarey“ die Gegengewalt provoziert habe (ebd.).

In der musikalischen Umsetzung von Graun sind die Worte ,Eroe/,Eroi (,Held $\%$,Helden') beziehungsweise ,Barbaro '/,Barbari' (,Barbar\%/,Barbaren'), die in den Arien meist als Anrede vorkommen, nicht spezifisch ausgeziert. ${ }^{29}$ Das Heroische als Haltung wird musikalisch anders vermittelt, nämlich durch fanfarenartigen Hornmotive und/oder in der langen, stark ausgezierten und mit prägnanten Rhythmen versehenen Koloratur auf „trionfar" in der Arie „Vegga, che alfin“ (I 3, 24) des Pilpatoe. ${ }^{30}$ Beide Mittel begegnen auch in Eupaforices Arie „L'onor del soglio“ (II 13, 102), in welcher der hier exorbitant verzierte Begriff „viltà" einerseits die Barbarei der Spanier, zugleich aber auch den heroischen Mut der Königin charakterisiert. ${ }^{31}$

29 Vgl. etwa die Passagen in der Partitur von Graun: Montezuma (Anm. 5), 78-79, wo im Empfangschor die Bezeichnung „stranieri eroi“ noch als Ehrentitel für die fremden Gäste aus Spanien gebraucht wird. Dass „eroe“ für Graun kein Schlüsselwort für die musikalische Ausdeutung ist, zeigt auch die Arie von Narves „Vedrai fra queste mura“. Hier ist im B-Teil (57) „eroi“ auf eine kleine Floskel gelegt, diese dann aber chiastisch ausgetauscht.

30 Vgl. Graun: Montezuma (Anm. 5), 28-33, hier 29.

31 Ebd., 155-162. 
Der Schlüsselbegriff ,Barbaro' wird prägnant eingesetzt, um das ungastliche, feindliche Verhalten, aber auch die Zumutung Cortes' gegenüber Montezumas Braut zu kennzeichnen und zu verurteilen. Zwei Mal ist „Barbaro“ als „Devise“ hervorgehoben, das heißt als ein einzelnes Wort, das am Anfang einer Arie oder eines Arienteils isoliert steht und dann nach einem kurzen Zwischenspiel wiederholt wird: jeweils im Allegro-Teil einer Arie Montezumas (,Del mio destin tiranno" [II 7, 78]) als auch in einer Arie der Eupaforice („Barbaro, che mi sei fiero“ [II 9, 88]). ${ }^{32}$ Durch den Tempowechsel in beiden Arien, ein fortschrittliches Gestaltungselement, das Graun anwendet, um die konstrastierenden Affekte zu unterstreichen, wird das heftige „Barbaro“ hervorgehoben und als musikalisches Motiv durch denselben Rhythmus (punktierte Viertel, Achtel, Viertel) und dasselbe Intervall (Quartsprung nach unten) markiert.

\subsection{Typologische Heroisierung}

Vieles spricht dafür, dass Friedrich der Große mit seinem Montezuma die dramatische Probe aufs Exempel seiner Machiavelli-Widerlegung liefern wollte. Tatsächlich wird Cortes in Friedrichs Anti-Machiavel als Beispiel eines „Glücksritters“ genannt. ${ }^{33}$ Wie Friedrich „dem Laster die Decke der Tugend wegzureißen“ bemüht ist, ${ }^{34}$ so entlarvt sich in der Tragödie der Eroberer Cortes selbst als macchiavellistischen Erfolgspolitiker, während Montezuma zum idealen Fürsten nach Maßgabe von Friedrichs Anti-Machiavel stilisiert wird. Montezuma ist wie „ein liebender Vater“ zu seinem Volk, bagatellisiert dies selbst aber als „,[s]eine Pflicht“ (I 1, 14-15), ist freigiebig, ${ }^{35}$ sorgt sich um das Wohl seiner Untertanen (Kap. 17). Vor allem aber ist er ehrlich und ,verstellt ${ }^{\varsigma}$ sich nicht, wie es Cortes im Sinne Machiavellis praktiziert, „dem Lehrmeister der Tyrannen und der Verstellungskunst“ (Kap. 18). Und schließlich willigt Montezuma erst in einen Krieg gegen die Spanier ein, als es das einzige, und, wie Friedrich betont, „rechtmässig[e]“ Mittel ist, „einen widerrechtlichen Angriff zu steuren, wohlbegründete Rechte zu erhalten, die Freyheit der Welt in Sicherheit zu stellen, und der Ehrsüchtigen Gewaltthätigkeiten und Unterdrückungen zu vermeiden“. 36

Neben dieser antimachiavellistischen Heroisierung entspricht die Figur des Montezuma aber noch einem zweiten typologischen Muster, dem des Märtyrers in der tragischen Märtyrer-Tyrannen-Konstellation. Den Protagonisten zeichnet eine

32 Ebd., 121 sowie 134.

[Friedrich II.:] Anti-Machiavel oder Versuch ein Critik über Machiavels Regierungskunst eines Fürsten [Anti Machiavel, hg. von Voltaire, 1740, dt.]. Frankfurt und Leipzig 1745, 205-397, besonders Kap. 25, 368-381, hier 377: „Sogar einem Fernando Cortez mußten die innerlichen Kriege der Amerikaner zur Bezwingung Mexikos behilflich sein“.

34 Ebd., besonders Kap. 26, hier 382.

35 Ebd., besonders Kap. 16, hier 305: „Ich kenne keinen Helden, der es [freigebig] nicht gewesen wäre".

36 Ebd., besonders Kap. 26, hier 392. 
Arglosigkeit aus, die der raffinierten Strategie des „Tyrannen“ Cortes nicht gewachsen ist. Zwar reagiert Montezuma auf seine Verhaftung mit heftigen Vorwürfen, doch er entpersonalisiert seinen Zorn zum "Schrecken [s]eines tyrannischen | Schicksals“ (II 7, 79) und „erschreckliche[m] Schicksal“ (III 1, 105). Gerade in dem pathetischen Accompagnato-Monolog der Gefängnisszene III 1 zeigt Montezuma, wenn er über das wechselnde Glück reflektiert und sich selbst zur „Apathía“ mahnt, eine stoisch-heroische Größe: „Laßt uns mit Gedult ein Uebel ertragen, | Das wir nicht ändern können! [...] So laßt uns, ohne über die Vorsicht Klage zu führen, | Getrost unserem Schicksal entgegen gehen“ (III 1, 109). Gerade in seiner Schlussarie, in der er seine Todesbereitschaft gelassen verkündet, gewinnt Montezuma die heroischen Züge eines - epikureischen - Märtyrers: „Ich überliefere ohne Furcht | Dem Schooße der Natur eine reine Seele, | Und meinen Körper den Elementen, | Denen er seine Geburth zu dancken hat" (III 5, 135). ${ }^{37}$

Beruht das Heldentum Montezumas zum einen auf der Kontrastfigur Cortes, zum andern auf seinem monologisch verbürgten Stoizismus, so kommt zum dritten die heroische Liebe zwischen Montezuma und Eupaforice hinzu. Während Montezuma seinen politischen Handlungsspielraum zunehmend einbüßt, übernimmt seine Braut Eupaforice sukzessive die Lenkung der Geschehnisse. Ist sie am Anfang nur treu liebende, besorgte Ratgeberin, wandelt sie sich im Verlauf des Stücks zur eigentlichen Heroine. Sie widersteht Cortes' erpresserischem Heiratsantrag, besticht die Gefängniswächter, um zu ihrem Geliebten zu gelangen, wagt einen Fluchtversuch, entwickelt den Plan einer militärisch-politischen Gegenoffensive und verhindert, indem sie Stadt und Palast in Brand setzt, dass dem Sieger Untertanen und Gold zur leichten Beute werden. Vor allem aber wird sie zur bestimmenden Figur der Tragödie durch ihren heroischen Selbstmord, den sie selbstbestimmt als demonstrativen Liebestod inszeniert. Selbst Montezuma überrascht die Tatkraft seiner Braut: „Ich hätte niemahls geglaubt, daß du mit | Den vielen Tugenden, die ich in dir verehrt habe, | Noch so vielen Muth verbändest" (III 2, 117). Die dynamische Konzeption der Eupaforice lobte schon Friedrichs Schwester Wilhelmine nach der Lektüre des Libretto-Entwurfs: „Das schöne Geschlecht ist Dir viel Dank schuldig; denn es kommt in Deiner Oper glänzend weg. Die Königin von Tlascala ist eine echte Heldin ohne Schwäche, voller Standhaftigkeit und um Mittel nie verlegen." ${ }^{38}$ Und Friedrichs italienischer Librettist lobt in seiner Leservorrede die Virago als die heimliche Heldin:

37 Eine ähnlich stoische Haltung gegenüber dem Wandel des Glücks zeigt Eupaforice in der Schlussszene des II. Akts („Die Tapfern können allein das Glück ändern“ [II 13, 101]).

38 Wilhelmine von Bayreuth an Friedrich II., 24. Mai 1754 (Anm. 14), 269-270. Eine analoge Figurenkonstellation prägt bereits die Oper Coriolano, in der in Gestalt der Volumnia eine selbstbewusste Frauenfigur einem eher wankelmütigen Titelhelden zur Seite gestellt ist. Vgl. Peter Schleuning: ,Ich habe den Namen gefunden, nämlich Montezuma': Die Berliner Hofopern Coriolano und Montezuma, entworfen von Friedrich II. von Preußen, komponiert von Carl Heinrich Graun. In: Klaus Hortschansky (Hg.): Traditionen - Neuansätze: Für Anna Amalie Abert (1906-1996). Tutzing 1997, 493-518. 
Je ne feray mention que du Role d'Eupaforice, qui es tun [sic!] de plus intéressans de la Piéce. On trouve dans cette Princesse un de ces Caractéres, qui s'sttachent tous les coeurs. Elle ne cesse de donner à Montezuma les plus sages conseils pour le tirer de la fatale securité: Elle brave la fureur et l'inhumanité de ses Tyrans; Elle entreprend tout pour rompre les fers de son Epoux, et pour le vanger: Quel courage, quelle intrépidité! ${ }^{39}$

\section{Fazit}

In Friedrichs Tragödie Montezuma werden der exotische Protagonist und mehr noch seine Gefährtin zu heroischen Figuren überhöht. Zu dieser Heroisierung tragen neben einem doppelten Perspektivismus, der die arglose Großmut der indigenen Gruppe mit der Heimtücke der Eroberer kontrastiert, typologische Charakterisierungstechniken bei: So wird Montezuma gegen die machiavellistische Kontrastfigur Cortes zum aufgeklärten Fürsten idealisiert und im tragischen Modell von Märtyrer - Tyrann zum stoischen Märtyrer erhöht. Zugleich wird seine Braut Eupaforice sukzessive zur Virago heroisiert und gewinnt durch ihren Freitod antike Größe. Damit repräsentiert in Friedrichs Montezuma die Neue Welt die humanistischen Werte der europäischen Antike, während die spanischen Konquistadoren zu skrupellosen, ehrgeizigen und habgierigen Machtpolitikern enthumanisiert werden, die mit der katholischen Religion einen brutalen Eroberungskrieg camouflieren.

\section{Abbildungsnachweise}

Abb. 1: Ruth Müller-Lindenberg (Hg.): Friedrichs Montezuma. Macht und Sinne in der preußischen Hofoper (26.1.-24.6.2012). Publikation zur Sonderausstellung im Musikinstrumenten-Museum des Staatlichen Instituts für Musikforschung Preußischer Kulturbesitz. Berlin 2012, 108.

Abb. 2: $\quad$ Rolf Badenhausen (Hg.): Die Bildbestände der Theatersammlung Louis Schneider im Museum der Preußischen Staatstheater Berlin. Systematischer Katalog. Berlin 1938.

Abb. 3: $\quad$ Ruth Müller-Lindenberg (Hg.): Friedrichs Montezuma. Macht und Sinne in der preußischen Hofoper (26.1.-24.6.2012). Publikation zur Sonderausstellung im Musikinstrumenten-Museum des Staatlichen Instituts für Musikforschung Preußischer Kulturbesitz. Berlin 2012, 55.

Abb. 4: Carl Heinrich Graun: Montezuma, hg. von Albert Mayer-Reinach (Denkmäler deutscher Tonkunst 1, 15). Wiesbaden und Graz 1958, 187.

39 Tagliazucchi: Au lecteur (Anm. 2), 4-5. 